\title{
Simulation of human gait with body weight support: benchmarking models and unloading strategies
}

\author{
Salil Apte ${ }^{3,1}$, Michiel Plooij $^{2}$ and Heike Vallery ${ }^{1,4^{*}}$
}

\begin{abstract}
Background: Gait training with partial body weight support (BWS) has become an established rehabilitation technique. Besides passive unloading mechanisms such as springs or counterweights, also active systems that allow rendering constant or modulated vertical forces have been proposed. However, only pilot studies have been conducted to compare different unloading or modulation strategies, as conducting experimental studies is costly and time-consuming. Simulation models that predict the influence of unloading force on human walking may help select the most promising candidates for further evaluation. However, the reliability of simulation results depends on the chosen gait model. The purpose of this paper is two-fold: First, using human experimental data, we evaluate the accuracy of some of the most prevalent gait models in replicating human walking under the influence of Constant-Force BWS: The Simplest Walking model (SW), the Spring-Loaded Inverted Pendulum model (SLIP) and the Muscle-Reflex (MR) gait model. Second, three realizations of BWS, based on Constant-Force (CF), Counterweight (CW) and Tuned-Spring (TS) approaches, are compared to each other in terms of their influence on gait parameters.

Methods: We conducted simulations in Matlab/Simulink to model the behaviour of each gait model under all three BWS conditions. Nine simulations were undertaken in total and gait parameter response was analysed in each case. Root mean square error (mrmse) w.r.t human data was used to compare the accuracy of gait models. The metrics of interest were spatiotemporal parameters and the vertical ground reaction force peaks. To scrutinize the BWS strategies, loss of dynamic similarity was calculated in terms of root mean square difference in gait dynamics ( $\Delta \mathrm{gd})$ with respect to the reference gait under zero unloading. These gait dynamics were characterized by a dimensionless number Modela-w.

Results: The SLIP model showed the lowest mrmse for 6 out of 8 gait parameters and for 1 other, the mrmse value were comparable to the MR model; SW model had the highest mrmse. Out of the three BWS strategies, Tuned-Spring strategies led to the lowest $\Delta \mathrm{gd}$ values.

Conclusions: The results of this work demonstrate the usefulness of gait models for BWS simulation and suggest the SLIP model to be more suitable for BWS simulations than the Simplest Walker and the Muscle-reflex models. Further, the Tuned-Spring approach appears to cause less distortions to the gait pattern than the more established Counterweight and Constant-Force approaches and merits experimental verification.
\end{abstract}

Keywords: Body weight support, Gait models, Human locomotion, Gait rehabilitation

*Correspondence: H.Vallery@tudelft.nl

'Department of Biomechanical Engineering, Delft University of Technology, Mekelweg 2, 2628 CD Delft, Netherlands

${ }^{4}$ Department of Rehabilitation Medicine, Erasmus MC, Postbus 2040, 3000 CA

Rotterdam, Netherlands

Full list of author information is available at the end of the article

(c) The Author(s). 2020 Open Access This article is licensed under a Creative Commons Attribution 4.0 International License, which permits use, sharing, adaptation, distribution and reproduction in any medium or format, as long as you give appropriate credit to the original author(s) and the source, provide a link to the Creative Commons licence, and indicate if changes were made. The images or other third party material in this article are included in the article's Creative Commons licence, unless indicated otherwise in a credit line to the material. If material is not included in the article's Creative Commons licence and your intended use is not permitted by statutory regulation or exceeds the permitted use, you will need to obtain permission directly from the copyright holder. To view a copy of this licence, visit http://creativecommons.org/licenses/by/4.0/. The Creative Commons Public Domain Dedication waiver (http://creativecommons.org/publicdomain/zero/1.0/) applies to the data made available in this article, unless otherwise stated in a credit line to the data. 


\section{Introduction}

Body weight supported training (BWST) is a common gait rehabilitation technique for individuals suffering from neurological impairment due to stroke, spinal cord injury, Parkinson's disease, etc. During BWST, a certain amount of the user's body weight is supported by a suspension system, typically through a harness [1]. Thereby, body weight support (BWS) systems allow therapists to provide gait rehabilitation training, without the need of providing complete physical assistance [2]. After undergoing BWST, individuals with neurological impairments have shown improvement in balance, motor function and overall locomotion [3-8]. In addition to these benefits, BWST can lead to improved psychological well-being, enhanced muscle mass and better cardiovascular health [9].

A BWS system is typically composed of an apparatus which provides the user with an unloading force when walking overground or on a treadmill $[10,11]$. The main purpose of providing a constant unloading force is to partially reduce gravity. The notion that constant force is the best solution for partial BWS has been dominating the field of BWS systems [12], and led to complex mechanical designs such as the Lokolift [10], the Zero-G [13], etc. These devices use active control in order to render a constant force. Note that this is still different from actual simulated gravity because the load is applied only to the upper body (distributed via the harness), and not in a distributed way on each single body segment [14]. Accurate investigations for swing phase therefore generally require set-ups similar to a parabolic space flight $[15,16]$, which are inconvenient to reproduce.

Passive, and more low-cost BWS realizations for providing constant unloading force typically constitute the use of an appropriately heavy counterweight or an elastic element such as a spring with specific pretension. While these devices provide constant unloading force in static conditions, the vertical movement of the center of mass (COM) of the user during locomotion leads to a vertical motion of the counterweight or the end-point of the elastic element. This results in the deviation of the unloading force from the set (constant) magnitude and thus these device are generally considered inferior to actuated, closed-loop controlled systems [10]. However, there may still be unexploited potential in such passive realizations. Particularly, it could be possible that a simple elastic support may even bring gait dynamics closer to unsupported gait than an actively rendered constant force, following the hypothesis stated in [17].

One way to predict the efficacy of existing and new BWS designs and modulation strategies is by simulating their influence on the locomotion of existing gait models. This can improve the efficiency of the design process by speeding up the iteration steps and reducing or postponing the need for hardware prototypes and experiments with human subjects. Examples of such an approach are the studies by Glauser et al., Ma et al. and Lu et al. [18-20]. These examples, however, show that there is a wide range of gait models currently being used for such a simulation and they range from the simplest (mass-spring-damper system) to the most complex musculoskeletal models.

The first goal of this research is to investigate the suitability of gait models for BWS simulation through a comparison with experimentally-obtained gait parameter data. Three prominent biomechanical gait models from the literature were simulated in the sagittal plane with BWS, and trends for gait parameters were documented. The three gait models (Fig. 1), in increasing order of complexity are: (1) Simplest Walking (SW) model , (2) Spring Loaded Inverted Pendulum (SLIP) model and (3) Muscle-reflex (MR) model [21-23].

The second goal is to compare the effect of three fundamental BWS strategies on human gait: (1) Constant-Force (CF): which emulates a constant vertical unloading force (2) Counterweight (CW): where a vertically moving counterweight is used to provide the unloading force and (3) Tuned-Spring (TS): where an elastic element (spring) with specifically tuned stiffness generates the unloading force. The latter two strategies are seemingly 'imperfect' realizations to achieve constant vertical support. By comparing their influence on gait parameters to that of an ideal constant unloading force, we aim to explore whether these imperfections are detrimental to the goals of BWS or even provide unexplored benefits for improving BWS design.

In the elastic BWS, the motion of the attachment point affects the deflection of the spring, thus causing variations in unloading force. The Tuned-Spring BWS system is based on the hypothesis [17] that such a variation is desirable and more beneficial than a constant force, because it maintains the dynamic similarity of gait despite unloading. While a constant unloading force partially compensates for the weight of the user, the inertia of the body still affects the dynamics of the gait. We hypothesize that if the unloading force can be tuned to compensate for both the gravitational and inertial forces, gait dynamics will be less modified. According to this hypothesis, and the associated design method presented in [17], the stiffness of the spring used for providing the unloading force can be tuned to compensate for inertial forces of the unloaded mass, thus enabling gait which is more dynamically similar to unsupported walking. This works for a periodic (ideally harmonic) movement of the body, and is quite robust to deviations.

Dynamic similarity [24, 25], based on the Froude number, has been previously used for investigating the effect of BWS on gait [26]. However, a recent work [27] suggested the Froude number alone to be inadequate and proposed a new metric called Modela-w. We thus use the change in Modela- $w$ caused by the different BWS 


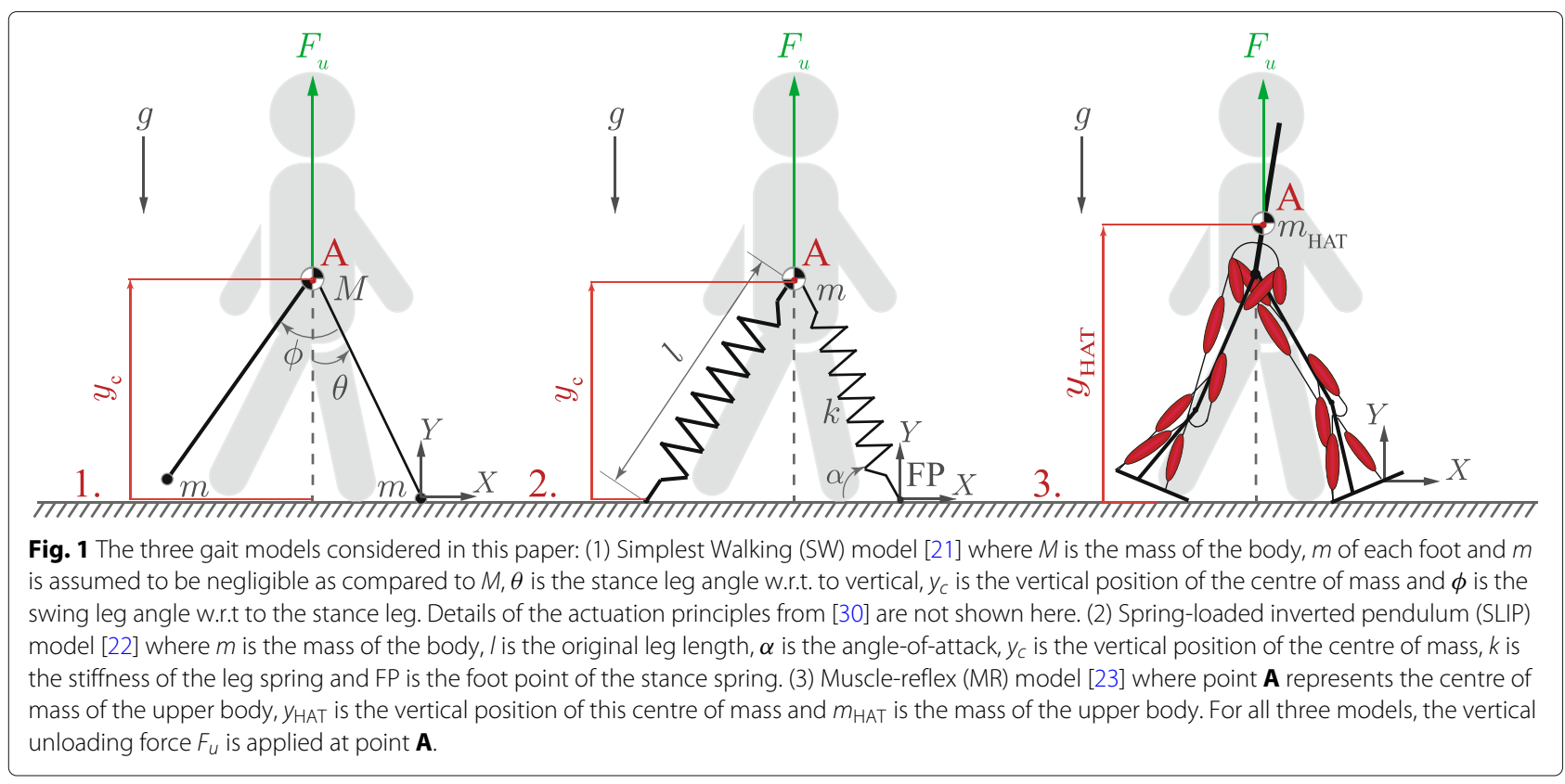

conditions to compare the three BWS strategies and test the aforementioned hypothesis.

Gait parameter trends produced by the simulations are compared with each other and with the human data trends (dataset available at [28]) obtained from an existing systematic review [12]. These trends resulted from the meta-analysis of around fifty existing studies measuring the influence of body weight support on gait parameters. While this meta-analysis presents data for both patients and healthy subjects, only the latter group is considered for comparison in the present study. Results used for benchmarking the gait models and comparing the BWS strategies are presented in "Comparison of gait models" subsection (Fig. 3, 4, and Table 2) and "Comparison of BWS strategies" subsection (Figs. 5, 4, and Table 4) respectively.

\section{Methods}

\section{Selection of gait models}

The scope of this research is limited to 2D gait models since all the gait characteristics of interest, i.e. those studied in [12], can be investigated using 2D models. These characteristics are the gait spatio-temporal parameters and the vertical ground reaction forces; the secondary ones are leg joint range of motion, joint moments, anteroposterior ground reaction forces, and leg muscle activities. These gait parameters are relevant because they have been extensively investigated in previous studies on the influence of BWS and used for designing and testing BWS systems [12]. Four gait models are particularly prominent in literature: (1) Linear inverted pendulum model (LIPM) [29], (2) Simplest walking (SW) model [21] (actuated on the basis of the principles suggested in [30]), (3) Spring-loaded inverted pendulum (SLIP) model [22], and (4) Muscle-reflex (MR) gait model [23]. The LIPM model, however, considers the centre of mass (COM) of the body to move in a straight horizontal line and thus the vertical movement of the COM needed to study the counterweight and tuned-spring BWS strategies is absent. As a result, this model was excluded from the selection of gait models. For the SW model, the foot mass is assumed to be negligible as compared to the body mass. The mechanical configuration and definition of variables for the three models are illustrated in Fig. 1.

\section{BWS strategies}

This section describes the three BWS strategies (Fig. 2) used for simulations, CF, CW and TS.

\section{Assumptions}

The simulations are based on five main assumptions (Fig. 2) - (1) the counterweight and the free end of the spring only move in the vertical (Y) direction, (2) pulley systems I and II, the ropes and the spring in Fig. 2 are massless, (3) the BWS system is frictionless and there is no net energy dissipated in the system, (4) the unloading force is applied at the center of mass (COM) of the upper body (head, arms and trunk, HAT), which in the cases of SW and SLIP models coincides with the body's overall center of mass, and (5) the pulley system I follows the attachment point $\mathbf{A}$ along the horizontal (X) direction and thus it is always perfectly overhead of the attachment point. This way, the BWS system does not apply any horizontal forces on the gait model nor does it add to the inertia of the model in horizontal direction. While the horizontal force components of the BWS system [31] can be important for determining the user's gait, we chose 


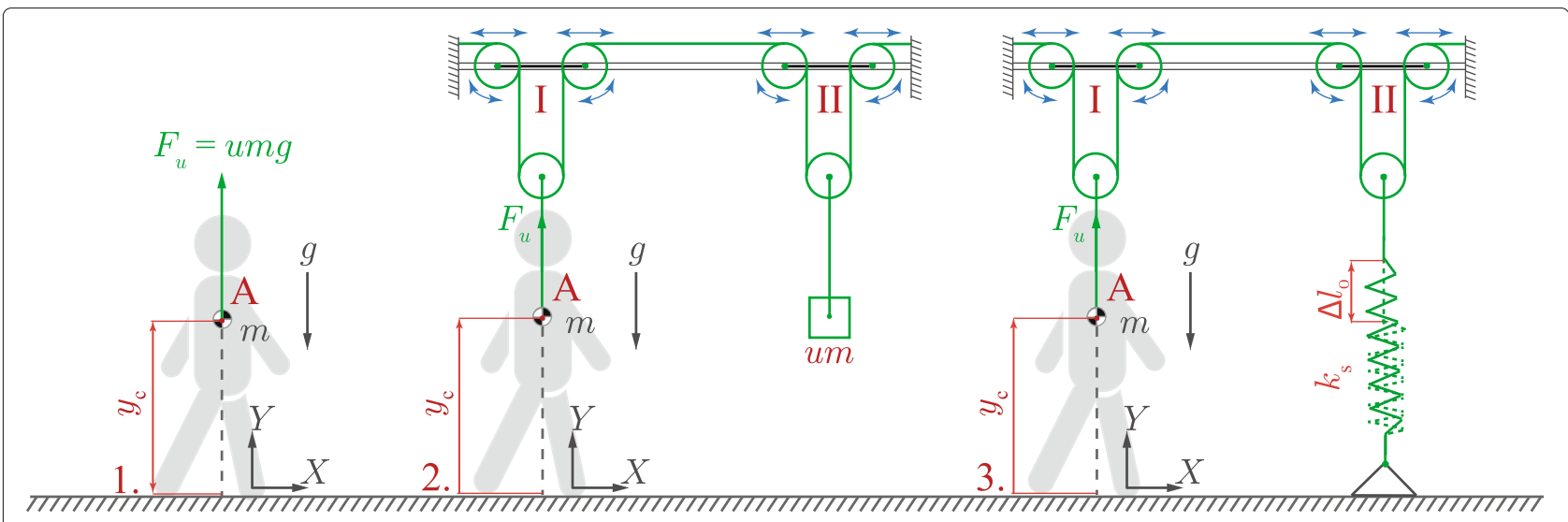

Fig. 2 The three BWS strategies: (1) Constant-Force (CF) (2) Counterweight (CW) system (3) Tuned-Spring (TS) system. Pulley systems I and II are designed such that the counterweight (of mass $u \cdot m$ ) and the free end of the spring only move vertically. The centre of the pulley system I is assumed to move horizontally such that force the $F_{u}$ is directed vertically upwards from point $\mathbf{A}$. All pulleys are massless and the system does not dissipate net energy. The coordinate $y_{c}$ is the vertical position of the centre of mass of the body, $u$ is the amount of body weight unloaded as a proportion of the actual body weight $m g, k_{s}$ is the stiffness of the spring, and $\Delta /_{0}$ is its initial elongation.

to focus solely on the influence of the vertical unloading force on the gait. Considering the $\%$ BWS supplied as $\beta$, the unloading coefficient $u$ as $u=\beta / 100$, total mass of the body as $m$ (Fig. 2) and the acceleration of gravity as $g$, the equations describing the three BWS strategies are presented below.

\section{Constant-Force system}

The Constant-Force (CF) BWS strategy consists of applying constant vertical force (Eq. 1) on the body. It can be considered as an ideal case of an unmodulated BWS [12]. Since the SW and SLIP models do not have distributed mass, the CF BWS strategy also emulates the effects of reduced gravity for these models [14]. However, this is not the case for the MR model [23] due to the presence of limb mass.

$$
F_{\mathrm{cf}}=u m g
$$

\section{Counterweight system}

The Counterweight (CW) BWS strategy is based on the use of a counterweight of mass $u m$ to provide $\beta \%$ of BWS. In the static case, this strategy leads to a constant unloading force $\left(F_{u}=u m g\right)$. However, the counterweight moves vertically as it follows the vertical motion of the attachment point A. Due to this motion, an additional inertial force $\left(u m \ddot{y}_{\mathrm{c}}\right)$ is generated, which disturbs the intended constant unloading force. Thus, instead of a constant unloading force, the force acting on the body is

$$
F_{\mathrm{cw}}=u m\left(g-\ddot{y}_{\mathrm{c}}\right)
$$

where $\ddot{y}_{\mathrm{c}}$ is the vertical acceleration of the attachment point $\mathbf{A}$ in upward direction.

\section{Tuned-Spring system}

An elastic element (spring), which can be considered massless as compared to a counterweight, can provide unloading force without the drawback of increasing inertial forces caused by the movement of a counterweight. As mentioned above, the spring can even further reduce inertial effects, which in effect means partially removing both gravitational and inertial forces acting on the human body simultaneously [17]. The spring stiffness $k_{\mathrm{s}}$ to achieve this needs to be tuned to:

$$
k_{\mathrm{s}}=u m \omega^{2} \text {, }
$$

where $\omega=2 \pi c$ and $c$ is the cadence (step-to-step frequency) of the walking model at $0 \%$ BWS. The initial deflection $\Delta l_{0}$ of the spring is chosen such that the unloading is equal to $u m g$ in the initial configuration of each model:

$$
\Delta l_{0}=\frac{u m g}{k_{\mathrm{s}}}=\frac{g}{\omega^{2}}
$$

The unloading force provided by the TS BWS strategy is:

$$
F_{\mathrm{ts}}=k_{\mathrm{s}}\left(y_{\mathrm{c} 0}-y_{\mathrm{c}}+\Delta l_{0}\right) \text {, }
$$

where $y_{\mathrm{c}}$ is the vertical position of point A at time $t$ and $y_{\mathrm{c} 0}$ is its average position during walking.

In case of the SW and SLIP models, $y_{\mathrm{c} 0}$ is considered to be the initial position of the model, since the difference between this and the average position is marginal, leading to a small $(<3 \%)$ difference in the intended and actual unloading levels.

For the MR model, choosing the initial vertical position (at $t=0$ ) of point $\mathrm{A}$ as $y_{\mathrm{c} 0}$ leads to higher unloading than desired, whereas the choice of mean vertical position at $0 \%$ unloading leads to a lower unloading force. Therefore, for this model, the value of $y_{\mathrm{c} 0}$ was estimated by a heavy first-order low-pass filter on the signal $y_{c}$ (Appendix A). 


\section{Model implementation}

\section{Simulation environments}

All gait models were obtained online [32-34] and were modified according to the equations presented below, in order to simulate the effect of all three BWS strategies. For the SW and SLIP models, the equations of motion were implemented in Matlab. In case of the MR model, Simscape blocks were created to emulate the Constant-Force (CF), Counterweight (CW) and Tuned-Spring (TS) BWS strategies, since the original Muscle-reflex gait model [23] was implemented in Simscape.

Only modified equations of motion for each model (Figs. 1 and 2) are presented here. The equations which are not affected by the BWS system are not presented and can be found in the original literature.

\section{BWS implementation for the simplest walking model}

The original equations of motion of the Simplest walking (SW) model are presented in [21]. The angle $\theta$ represents the stance leg angle w.r.t. the vertical and $\phi$ is the swing leg angle w.r.t. the stance leg. Following the original paper, time is scaled by $\sqrt{\frac{l}{g}}$ for all three BWS strategies. A hip spring with the dimensionless torsional stiffness $k_{\mathrm{f}}$ is used for actuation [30]. The 'foot' mass $m$ is assumed to be much smaller than the 'body' mass $M$, so $m / M \approx 0$.

Constant-Force: A term representing the constant vertical unloading force $\left(F_{u}=u M g\right)$ was added to the original equations [21], leading to:

$$
\begin{aligned}
& \ddot{\theta}=(1-u) \sin \theta \\
& \ddot{\phi}=\ddot{\theta}+\dot{\theta}^{2} \sin \phi+u \sin \theta \cos \phi-\cos \theta \sin \phi-k_{\mathrm{f}} \phi .
\end{aligned}
$$

Counterweight system: The mass of the counterweight is $u M$, where $M$ is the mass at the hip, so

$$
\begin{aligned}
\ddot{\theta}= & \frac{1-u}{1+u} \sin \theta \\
\ddot{\phi}= & \ddot{\theta}+\dot{\theta}^{2} \sin \phi-\cos \theta \sin \phi \\
& +\frac{2 u}{1+u} \sin \theta \cos \phi-k_{\mathrm{f}} \phi .
\end{aligned}
$$

Tuned-Spring system: Considering Eqs. (3-5) in "Methods" section, $y_{\mathrm{c}}=l \cos \theta$ to be the vertical position of point $\mathrm{A}$ at time $t$ and $y_{\mathrm{c} 0}=l$ at $t=0$, the EOM are:

$$
\begin{aligned}
\ddot{\theta}= & (1-u) \sin \theta+\frac{l}{g} \omega^{2} u(1-\cos \theta) \sin \theta \\
\ddot{\phi}= & \ddot{\theta}+\dot{\theta}^{2} \sin \phi-\cos \theta \sin \phi \\
& -u\left(1+\frac{l}{g} \omega^{2}(1-\cos \theta)\right) \sin \theta \cos \phi-k_{\mathrm{f}} \phi .
\end{aligned}
$$

\section{BWS implementation for the bipedal spring-loaded inverted pendulum model}

The gait cycle in the SLIP model, as given in the original paper [22] is divided into three phases - initial single limb stance (SLS) of the left leg, intermittent double-limb stance (DLS) and final single limb stance (SLS) of the right cycle. The equations for horizontal acceleration do not change since BWS is assumed to influence only the vertical motion. The modified equations of motion for the vertical motion of the COM depend on the chosen BWS strategy.

Constant-Force system: A term representing the constant vertical unloading force $\left(F_{u}=u M g\right)$ is added to the original equations [22], so:

$$
\begin{aligned}
\text { Initial SLS: } & m \ddot{y}_{\mathrm{c}}=P y_{\mathrm{c}}-m(1-u) g . \\
\text { DLS: } & m \ddot{y}_{\mathrm{c}}=P y_{\mathrm{c}}+Q y_{\mathrm{c}}-m(1-u) g . \\
\text { Final SLS: } & m \ddot{y}_{\mathrm{c}}=Q y_{\mathrm{c}}-m(1-u) g .
\end{aligned}
$$

Counterweight system: The mass of the counterweight is $u m$, where $m$ is the mass of the body, thus leading to:

$$
\begin{aligned}
\text { Initial SLS: } & m \ddot{y}_{\mathrm{c}}=P y_{\mathrm{c}}-m \frac{(1-u)}{1+u} g . \\
\text { DLS: } & m \ddot{y}_{\mathrm{c}}=P y_{\mathrm{c}}+Q y_{\mathrm{c}}-m \frac{(1-u)}{1+u} g . \\
\text { Final SLS: } & m \ddot{y}_{\mathrm{c}}=Q y_{\mathrm{c}}-m \frac{(1-u)}{1+u} g .
\end{aligned}
$$

Tuned-Spring system: Considering Eqs. (3-5) in "Methods" section, the resulting equations for the Tuned-Spring strategy are:

$$
\begin{aligned}
\text { Initial SLS: } & m \ddot{y}_{\mathrm{c}}=P y_{\mathrm{c}}-m g+F_{\mathrm{ts}} . \\
\text { DLS: } & m \ddot{y}_{\mathrm{c}}=P y_{\mathrm{c}}+Q y_{\mathrm{c}}-m g+F_{\mathrm{ts}} . \\
\text { Final SLS: } & m \ddot{y}_{\mathrm{c}}=Q y_{\mathrm{c}}-m g+F_{\mathrm{ts}},
\end{aligned}
$$

where $F_{\mathrm{ts}}$ is the unloading force provided by the TS BWS system (equation 5), $y_{c}$ represents the vertical position of the COM of the body. The terms $P$ and $Q$ are the same as those defined in [22]

$$
P=k\left(\frac{l_{0}}{\sqrt{x_{c}^{2}+y_{\mathrm{c}}^{2}}}-1\right) \quad \& \quad Q=k\left(\frac{l_{0}}{\sqrt{\left(d-x_{c}\right)^{2}+y_{\mathrm{c}}^{2}}}-1\right),
$$

where $d=\mathrm{FP}_{i+1, x_{c}}-\mathrm{FP}_{i, x_{c}}$, FP is the foot point of the stance spring, and $x_{c}$ is the horizontal position of the COM.

\section{BWS implementation for the muscle-reflex model}

The unloading force term for each BWS strategy were implemented as Simscape blocks according to the equations 1, 2 and 5, which present the unloading force for the CF, CW and TS BWS strategies respectively.

\section{Simulation protocol}

Each modified model was simulated with BWS ranging from $0 \%$ to $100 \%$, in $5 \%$ increments. The unloading force was applied at the center of mass of the body ( $\left.\mathrm{COM}_{\text {body }}\right)$ for all gait models and the COM of the upper body for 
the MR model (Appendix B). Initial pose for the SLIP and MR models is standing, at the instant before toe-off, while it is in the double stance after impact in case of the SW model. To test the model sensitivity to initial conditions, fifteen different starting gait speeds were used for simulation, ranging from $0.6185 \mathrm{~m} / \mathrm{s}$ to $1.6185 \mathrm{~m} / \mathrm{s}$ for the SLIP model (originally $1.1185 \mathrm{~m} / \mathrm{s}$ [22]) and $0.8 \mathrm{~m} / \mathrm{s}$ to $1.8 \mathrm{~m} / \mathrm{s}$ for the MR model (originally $1.3 \mathrm{~m} / \mathrm{s}$ [23]). Since the starting gait speed is not selectable for the SW model, starting step length was varied from 0.2167 to $1.2167 \mathrm{~m}$ (originally $0.7167 \mathrm{~m} \mathrm{[21]).} \mathrm{The} \mathrm{remaining} \mathrm{initial} \mathrm{condi-}$ tions and model parameters used in simulation are the same as those proposed in the original papers [21-23]. The highest percentage of BWS for which the model was able to achieve a walking gait for at least 20 steps was noted as the 'Maximum feasible BWS' $\left(\beta_{\max }\right)$ for each strategy. This $\beta_{\max }$ and the resultant average gait speed were used to understand the sensitivity of gait models to initial conditions.

Results for CF BWS strategy were selected for the comparison with experimental data since this data [28] was available only for Constant-Force BWS systems [12].

\section{Data analysis}

\section{Selection and analysis of gait parameters}

Relevant gait data was extracted for the starting gait speed or step length which was closest to the values proposed in the original papers and which led to the highest maximum feasible BWS. For each condition, the gait data was averaged over at least five strides in order to reduce the variability. The average step duration was considered as the inverse of cadence.

The proportion of each gait phase was computed with respect to the entire stride duration. The hip range of motion was calculated from the peak flexion angle following initial contact to the peak extension angle at terminal stance [35]. The knee range of motion was considered from the peak extension angle at terminal stance to the peak flexion angle at mid-swing. Peak joint torque values for flexion and extension were extracted from the torque patterns over a complete gait cycle, and indicated by negative and positive signs, respectively. The two peak values for the vertical ground reaction forces (GRF) and the extrema of the anteroposterior GRF over a single gait cycle were also computed. For muscle activity, the mean value over a complete gait cycle was considered. Some model-specific data analysis procedures were adopted, as listed below:

- The SW model was analyzed only for the gait spatio-temporal parameters like stride length, cadence, walking speed and the total stance phase. The model has an instantaneous double support phase, so only the total stance phase is considered.
Ground reaction forces (GRF) were not considered either, since they do not follow the characteristic pattern of anthropomorphic bipedal gait [36].

- The SLIP gait model was investigated for all gait spatio-temporal parameters and the vertical GRF.

- Since the MR gait model typically utilizes muscle groups, while the meta-analysis of experimental gait data [12] provides muscle activity data for individual muscles, the correspondence in Table 1 was used for comparing the results.

\section{Comparison of gait parameter response}

Suppose $P=\left[P_{0}, P_{5}, P_{10}, \ldots ., P_{\beta_{\max }}\right]$ represents the vector of values for a specific gait parameter at each unloading level up to the 'Maximum feasible BWS' $\left(\beta_{\max }\right)$, in increments of $5 \%$. This data was normalized by taking a ratio with the parameter value at $0 \%$ BWS, resulting in $P_{N}=P / P_{0}=\left[1, P_{5} / P_{0}, P_{10} / P_{0}, \ldots ., P_{\beta_{\max }} / P_{0}\right]$ The aim was to reduce variability in results and allow comparison of trends across gait models. By removing the dimensions attached to each parameter through normalizing, comparison across different gait parameters was possible. The data from the meta-analysis [12] was used as reference human data, $P_{H}=\left[1, P_{H 5}, P_{H 10}, \ldots ., P_{k}\right]$. Because this reference data is already normalized, the normalization procedure for the gait model parameters was necessary for comparison with human data.

To compare the gait models, for each gait parameter, the root mean square error with respect to the experimental data was calculated for overground and treadmill walking environments. Since it is used to compare the gait models, this root mean square error is referred to as mrmse. The mrmse (Eq. 22) was computed as a percentage of the gait parameter value at $0 \%$ BWS. The $0 \%$ BWS condition was not considered during mrmse calculation since the gait parameter data was normalized, such that the error at $0 \%$ BWS was always 0 . A lower value of the mrmse, so a better fit with the experimental data, means that the model is better suited to investigate the influence of BWS on that specific gait parameter. The comparison of gait models is based only on the mrmse values for the overground condition with a Constant-Force BWS system. The data considered for analysis ranged from $0 \%$ to $40 \%$ BWS, because at least two models could not achieve stable

Table 1 Muscle groups in MR gait model and corresponding muscles in experimental data

\begin{tabular}{ll}
\hline MR gait model & Experimental data \\
\hline 1. 'Vastus' muscle (VAS) / Quadriceps & Rectus femoris \\
2. Hamstring (HAM) & Biceps femoris \\
3. Gastrocnemius (GAS) & Lateral gastrocnemius \& \\
& Medial gastrocnemius \\
4. Tibialis anterior & Tibialis anterior
\end{tabular}


gait above $40 \%$. A missing data point indicates inability of the gait model and BWS strategy to produce a gait at that BWS level, which was penalized during the calculation of mrmse to reflect higher error values. $P_{N}$ was considered to be zero for this BWU level, since this can be considered as the highest deviation from the gait at $0 \%$ and thus it leads to the largest possible error for that unloading level. Human data indicates only a small influence of unloading force on gait parameters up to $30 \%$ BWS [12], which implies that the mrmse w.r.t. to the $0 \%$ BWS should also be small for the gait models, up to $30 \%$ unloading:

$$
\text { mrmse }=\sqrt{\frac{\sum_{k=1}^{8}\left(P_{H}(k)-P_{N}(k)\right)^{2}}{8}} \times 100 \%,
$$

where $\mathrm{k}$ is the index of the BWS levels, which are $k \cdot 5 \%$, thus ranging from 5 to $40 \%$.

In the context of BWS, the level of dynamic similarity between the unloaded walking task and the eventual task to be trained, namely walking without support, is crucial. In order to quantify this dynamic similarity, we first represented the gait dynamics for each condition by a dimensionless number, modela-w [27]:

$$
\text { modela-w }=\left(\frac{2 g l}{v^{2}}+\left(\frac{f l}{v}\right)^{2}\right)^{-1},
$$

where $g$ is the gravity, $f$ the frequency oscillation of the COM, $l$ the COM height which is leg length for SW and SLIP models, and $v$ is the forward speed. In the next step, we quantified the loss in dynamic similarity by calculating the combined root mean square difference of the modela$\mathrm{w}$ magnitude from $5 \%$ to $35 \%$ unloading with respect to the magnitude at $0 \%$ BWS for the SLIP and MR models For the SW model, it was calculated only from 5\% to $10 \%$ unloading for CF and TS strategies and only at 5\% for the $\mathrm{CW}$ strategy. To compare BWS strategies, we assumed that the strategies which lead to lower root mean square difference values are likely to distort gait dynamics less. This root mean square error is referred to as $\Delta \mathrm{gd}$ (Eq. 24) because it forms the basis for comparison of change in gait dynamics or the loss of dynamic similarity:

$\Delta \mathrm{gd}=\sqrt{\frac{\sum_{k=1}^{7}(\text { modela-w }(n)-\text { modela-w }(0))^{2}}{7}} \times 100 \%$,

where $k$ represents the BWS levels in the form of $k \cdot 5 \%$, ranging from $5 \%$ to $35 \%$.

\section{Results}

\section{Comparison of gait models}

The gait parameter values at different levels of ConstantForce BWS for each gait model are plotted in Fig. 3, along with the experimental data obtained from the metaanalysis [12] for healthy individuals walking in overground and treadmill environments. Gait parameters which were present only in one model, i.e the MR model, are included in Appendix C (Fig. 6). The mrmse for each model and the relevant gait parameters are presented in Table 2. Values for the treadmill condition are presented only for comparison with the overground condition for the same model and not between two models. Results for the sensitivity analysis are shown in Fig. 4.

\section{Simplest walking model}

The SW model had the highest mrmse values for all relevant gait parameters: stride length, cadence, walking speed, and total stance phase (Fig. 3 \& Table 2). The magnitude of mrmse was similar for overground and treadmill walking. Average gait speed showed low sensitivity (Fig. 4) to the magnitude of BWS but was relatively high for the initial step length; the sensitivity hardly changed for different BWS strategies.

\section{Spring-loaded inverted pendulum gait model}

The SLIP model had the lowest mrmse values for six out of eight paramaters, including stride length, cadence, walking speed, double limb stance, and vertical GRF (Fig. 3). For single limb stance phases, it showed a moderate mrmse value, comparable to the MR gait model, while for total stance phase, it was almost twice that of the MR model. Apart from the cadence, double limb stance, and vertical GRF peak I, the four remaining gait parameters had a higher mrmse value for overground walking. Average gait speed for SLIP model was highly sensitive (Fig. 4) to the initial speed for the CW BWS strategy while being relatively low for the other two strategies; it was low with respect to the level of BWS for all three BWS strategies.

\section{Muscle-reflex gait model}

The Muscle-reflex (MR) gait model was the only one which could be tested for almost all gait parameters mentioned in the meta-analysis [12]. Of the 23 gait parameters analyzed, this model had a mrmse of less than $10 \%$ for only eight characteristics (Table 2 and 5): total stance phase, double limb stance, vertical GRF peak I, hip joint ROM, knee flexion moment, ankle plantarflexion moment, gastrocnemius, and tibialis muscle activity. Except for single limb stance, and total stance phases, the MR model had higher mrmse values for other gait parameters than the SLIP model but lower than the SW model (Table 2).

However, the MR model had a high mrmse for knee and hip extension moments, anteroposterior GRF, ankle joint ROM and quadriceps muscle activity (Appendix C Fig. 6). Furthermore, for 8 gait parameters, the MR model showed lower mrmse values for the treadmill walking condition than for the overground condition. These parameters included total stance, single limb stance, vertical GRF peak II, hip moments, ankle plantarflexion moment, anteroposterior GRF peak I and tibialis anterior muscle activity (Fig. 3, Table 2, 5, and Appendix C - Fig. 6). Sensitivity of the average gait speed (Fig. 4) was low 


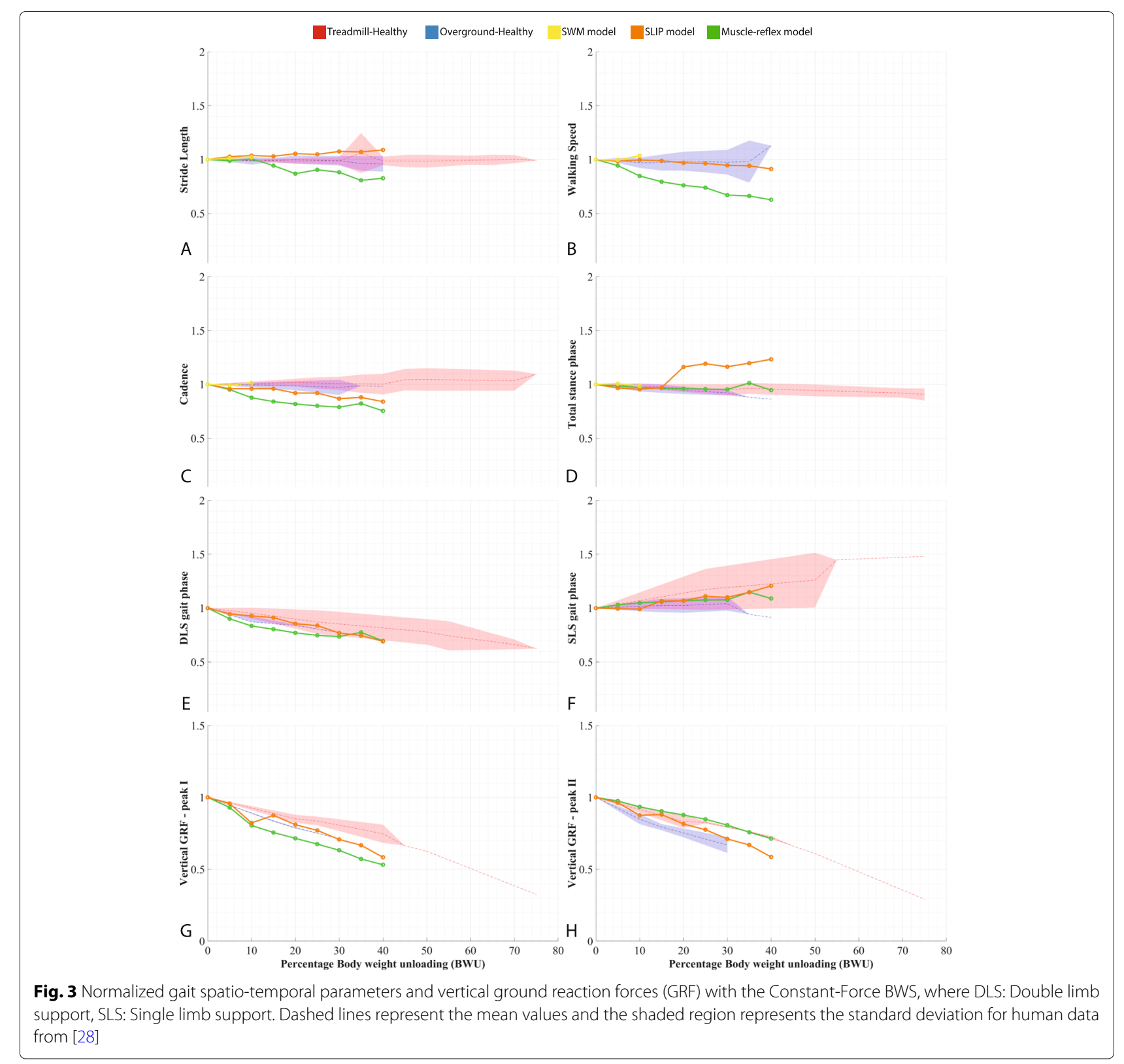

towards the initial speed and high for the level of BWS, for all three BWS strategies.

\section{Comparison of BWS strategies}

The highest feasible BWS $\left(\beta_{\max }\right)$ values for the three models and the BWS strategies are presented in Table 3. The trends for modela-w values are plotted in Fig. 5 and the range of feasible BWS levels across different initial gait speeds and step lengths (SW model) are plotted in Fig. 4. The range here refers to the difference between the minimum (not necessarily $0 \%$ ) and maximum feasible BWS levels. The change in gait dynamics $(\Delta \mathrm{gd})$ or the loss of dynamic similarity for each model in each BWS strategy is presented in Table 4.

\section{Counterweight BWS}

The Counterweight (CW) BWS strategy typically led to the lowest $\beta_{\max }$ values (Table 3) for the SW and MR models, and lowest feasible BWS ranges (Fig. 4) across all three gait models. This is reflected in the high $\Delta \mathrm{gd}$ values (Table 4) for all models and a stronger change in modela-w values (Fig. 5) for the SW and SLIP models. Sensitivity of the $\beta_{\max }$ to initial conditions was highest in case of CW BWS strategy (Fig. 4), especially for the SLIP model.

\section{Constant-force BWS}

With regards to the SW and MR gait models, the constantforce (CF) BWS strategy typically produced a higher $\beta_{\max }$ 
Table 2 Summary of the gait parameters results for the three gait models

\begin{tabular}{|c|c|c|c|c|c|c|}
\hline \multicolumn{4}{|c|}{ Root mean square error (mrmse) values } & \multirow{2}{*}{\multicolumn{3}{|c|}{ Treadmill (TM) }} \\
\hline \multirow{2}{*}{$\begin{array}{l}\text { Experimental } \\
\text { data } \\
\text { Gait model }\end{array}$} & \multicolumn{3}{|c|}{ Overground (OG) } & & & \\
\hline & sw & SLIP & MR & SW & SLIP & $\overline{M R}$ \\
\hline Gait parameter & $\%$ & $\%$ & $\%$ & $\%$ & $\%$ & $\%$ \\
\hline 01. Stride length & 86.61 & 7.60 & 10.10 & 86.61 & 6.22 & 12.46 \\
\hline 02. Cadence & 86.61 & 8.27 & 16.30 & 86.60 & 10.45 & 16.30 \\
\hline $\begin{array}{l}\text { 03. Walking } \\
\text { speed }\end{array}$ & 86.63 & 7.93 & 27.45 & - & & \\
\hline $\begin{array}{l}\text { 04. Gait phases - } \\
\text { Stance }\end{array}$ & 86.61 & 11.40 & 5.74 & 86.60 & 6.94 & 1.92 \\
\hline $\begin{array}{l}\text { 05. Gait phases - } \\
\text { Double limb } \\
\text { stance }\end{array}$ & - & 3.60 & 4.94 & - & 6.64 & 11.07 \\
\hline $\begin{array}{l}\text { 06. Gait phases - } \\
\text { Single limb } \\
\text { stance }\end{array}$ & - & 13.31 & 10.07 & - & 6.20 & 8.09 \\
\hline $\begin{array}{l}\text { 07. Vertical GRF } \\
\text { peak - I }\end{array}$ & - & 3.49 & 7.25 & - & 6.67 & 13.41 \\
\hline $\begin{array}{l}\text { 08. Vertical GRF } \\
\text { peak - II }\end{array}$ & - & 5.74 & 11.26 & - & 4.39 & 2.64 \\
\hline
\end{tabular}

Root mean square error (mrmse) values for 5-40\% Constant-Force BWS levels w.r.t the experimental data for overground (OG) and treadmill (TM) environments are presented here. Lowest mrmse values for each gait parameter are indicated in italics and green

value than $\mathrm{CW}$ BWS and a lower value than tunedspring (TS) BWS (Table 3), and vice-versa for $\Delta$ gd values (Table 4) for all three models. It showed a similar trend for the range of feasible BWS levels (Fig. 4) and the modela$\mathrm{w}$ values(Fig. 5). Finally, the sensitivity of $\beta_{\max }$ to initial conditions was lower than the $\mathrm{CW}$ strategy and higher than TS.

\section{Tuned-Spring BWS}

The Tuned-Spring (TS) produced the highest $\beta_{\max }$ values among all three BWS strategies for SW and MR models, while leading to high but not the highest values for the SLIP model. It also led to lowest change in gait dynamics (Table 4 and Fig. 5) across all models. Further, the $\beta_{\max }$ values were least sensitive to the initial condition (Fig. 4) for the TS BWS strategy in case of the SLIP and MR models.

\section{Discussion}

\section{Comparison of gait models}

The SW model showed the highest mrmse values for all four gait parameters (Table 2), namely stride length, cadence, walking speed, and stance phase duration. While Fig. 3 does not reflect such high mrmse values $(\approx 50 \%)$, these values are expected due to the penalization process explained in the earlier
"Comparison of gait parameter response" section. Onwards from 15\% BWS, the SLIP model presented a sudden increase in the proportion of single limb stance phase and consequently for total stance phase, relative to human data and the MR gait model, which led to a high m-rmse. This phenomenon can be attributed to the stabilization effect of the unloading force during the single limb stance. This effect was more pronounced in the SLIP model than in the MR model, as the MR model is comparatively more robust to disturbances [23]. However, for other parameters, the SLIP model showed the best performance out of the three models, despite its relative simplicity. Typically, the aim of active BWS is to enable the magnitude of spatio-temporal parameters to be similar to the values during unsupported walking or to retain the M-shape of the vertical GRF [37, 38]. These gait parameters are present in the SLIP model, and they change in similar ways as in the experimental data. This indicates that the SLIP model can likely be used effectively to simulate the effects of modulated BWS on gait spatio-temporal parameters and ground-contact interactions.

While the SW and MR models add energy to the system to maintain stable walking, the SLIP model does not. This feature of the SLIP model might be one of the reasons for its good performance and is worthy of further investigation. The SLIP model was only tested up to $40 \%$ BWS since the MR model could not achieve a walking gait beyond that level and thus no data was available for comparison of models. Thus, the accuracy of the SLIP model for higher BWS levels could not be investigated.

In case of the MR model, the unloading force produced an additional torque about the hip joint which needed to be counter-balanced by muscle forces. This led to an increase in the hip flexion moment and a decrease in the hip extension moment and subsequently affected the knee extension moment as well (Fig. 6). While analyzing the data, it was noted that the peak knee extension torque shifted temporally from just after initial contact to just before toe-off at $10 \%$ BWS. This temporal change in torque peak led to a sharp drop in knee extension moment magnitude, as seen in Fig. 6E. This could explain the sizable deviations from the human data for the hip and knee joint moments and thus the high mrmse (Table 5). While the ankle plantarflexion moment in the MR model was less affected by BWS than in humans, ankle angle ROM dropped almost 20\% lower than the human data. This reduction in ankle ROM, in combination with lack of change in ankle plantarflexion moment, led to a higher reduction in the forward push-off force (anteroposterior GRF peak II - Fig. 6I) and a lower reduction in the vertical push-off force (vertical GRF peak II - Fig. 3H) as compared to human data. In case of the muscle activities, muscle groups in the MR gait model were compared to 


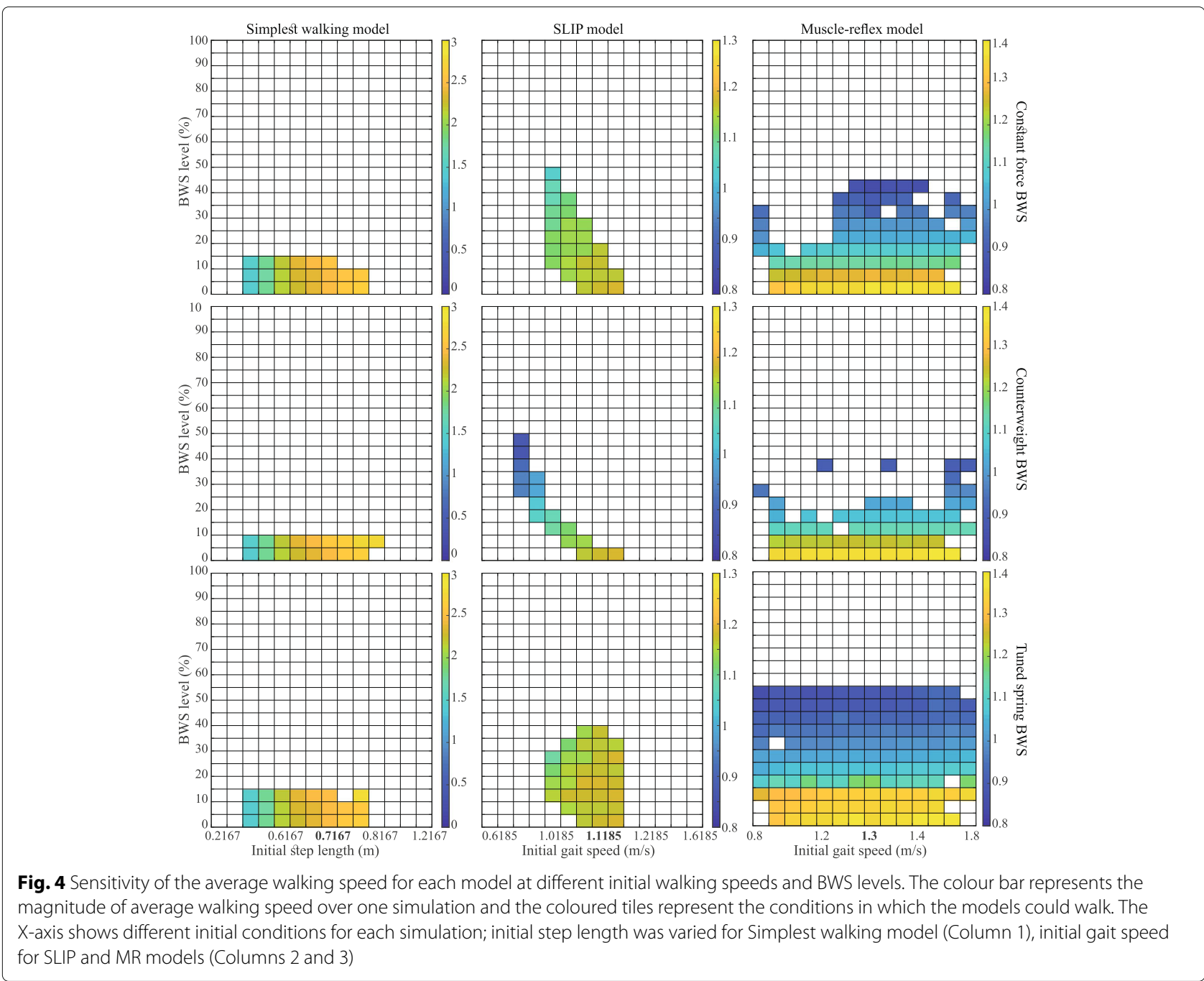

individual muscles in the experimental data (Table 1). While the muscle activities of the individual muscles are correlated to the muscle groups [39], the MR model showed high mrmse values ( $>10 \%$ ) for all muscles (Table 5) except the Lateral Gastrocnemius (LG) muscle.

The muscle reflexes and initial conditions for the MR model were not optimized for BWS, which might partially explain its lower performance. While an optimization would likely have led to a higher value of $\beta_{\max }$, the non-optimized model still yields comparatively high $\beta_{\max }$ values (Table 3 ). However, hand-tuning the model to suit every modulated BWS level would require extensive human data from

Table 3 Maximum value of BWS ( $\beta_{\max }$ ) at which the model still achieved a walking gait

\begin{tabular}{llll}
\hline & Constant-Force & Counterweight & Tuned-Spring \\
\hline SW model & 10 & 5 & 10 \\
SLIP model & 45 & 45 & 35 \\
MR model & 40 & 35 & 50 \\
\hline
\end{tabular}

experiments with modulated BWS, and obtaining this data is difficult. While optimization algorithms can be used to tune the model parameters [40], designing an appropriate cost function is difficult. Yet, this model could still be useful in certain scenarios, wherein the muscle reflexes could be tuned to emulate the pathological muscle function in individuals with neuromuscular disorders. Further, the MR model can also be used to optimize the BWST for biomechanical outcomes such as joint loading, investigating the impact of different BWS attachment points on the upper body, etc. Finally, the $\beta_{\max }$ values for the MR model are less sensitive to the initial gait speed than the SLIP model. Thus, the MR model offers a more robust alternative to the SLIP model for simulating a wider variety of initial conditions, albeit with a lower accuracy.

The starting conditions in the simulation for each model were selected based on their ability to produce the maximum feasible body weight support level. While this led to the comparison of models under differing simulation 

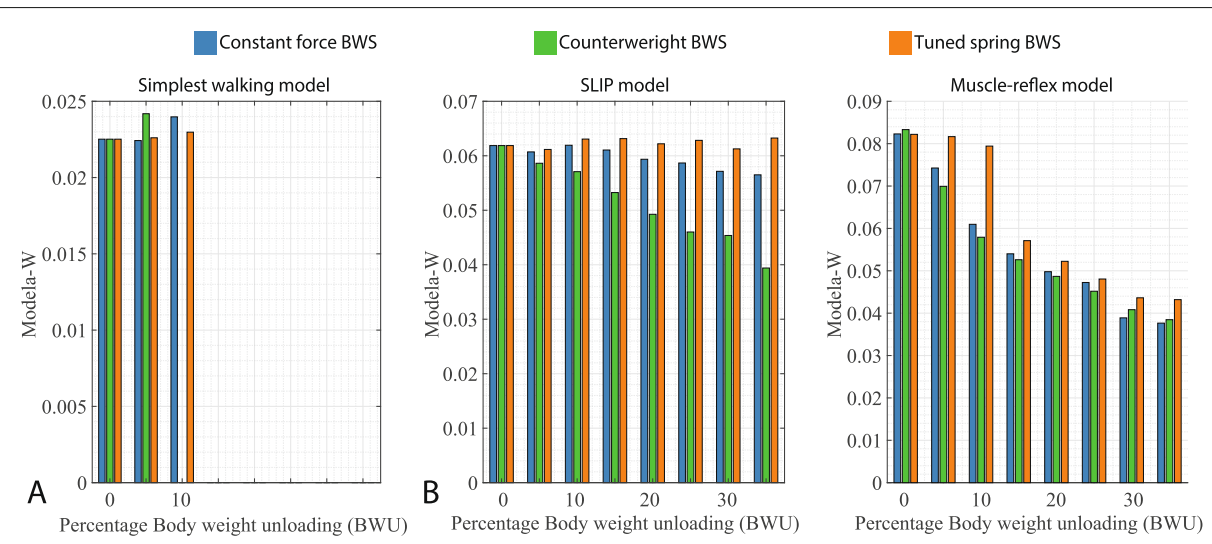

Fig. 5 Modela-w values with respect to increasing BWS levels for all three models and BWS strategies. A stronger change in Modela-w values represents a stronger change in gait dynamics and thus a higher loss in dynamic similarity

conditions, the evaluation concerned only the ability of the models to reproduce the gait parameter trends in response to different levels of BWS, but not the ability of the models to reproduce gait without BWS. Therefore, there remains a absolute bias error for all models. These absolute errors have been evaluated in [21-23]. Further, the maximum feasible BWS support for the SLIP and MR models is sensitive to the initial gait speed for the CF BWS condition, which makes it pertinent to select the appropriate initial speed.

\section{Performance of the BWS strategies}

The three BWS strategies evoked substantially different responses, especially in the SLIP and MR models. The $\beta_{\max }$ values were typically highest for the TS BWS strategy and lowest for the CW BWS strategy (Table 3). This highlights the importance of considering inertia in the design of BWS systems. Fig. 5 shows that TS BWS had a lower influence on gait dynamics than the other two strategies. It also led to a more consistent range (Fig. 4) of feasible BWS across all initial conditions. In case of SLIP and MR models, the TS BWS produced the lowest $\Delta$ gd values for all gait models (Table 4).

High $\beta_{\max }$ and low $\Delta$ gd values for the TS BWS strategy support the hypothesis that a spring-based BWS can enable gait which is more similar to unsupported walking [17]. Ideally, dynamic similarity in this case means that

Table 4 Change in the dimensionless constant Modela-w for all models and under each BWS strategy

\begin{tabular}{llll}
\hline & Constant-Force & Counterweight & Tuned-Spring \\
\hline SW model & 2.30 & 2.62 & 0.74 \\
SLIP model & 4.77 & 20.57 & 1.50 \\
MR model & 37.16 & 38.52 & 32.50
\end{tabular}

Root mean square of the difference between the magnitude of Modela-w from 5\% to $35 \%$ BWS levels with respect to the magnitude at 0\% BWS is shown here. This metric $(\Delta g d)$ is calculated as percentage and the least change in Modela-w i.e. the highest dynamic similarity condition is indicated in green and italics also the neural control strategy does not need to be changed when walking under the influence of body weight support. This makes a case for experimental evaluation, and questions the predominant paradigm of perfectly controlled constant unloading forces as being preferable to simple elastic support. Unloading force rendered by the TS BWS depends on the initial zero-deflection set-point of the spring and thus, it is important to consider an appropriate value for the initial set-point during the experimental evaluation of this strategy. Active BWS systems like the ZeroG [13], the FLOAT [41], the RYSEN [42], etc. could measure and slowly adjust to the average position during walking in real time. For simpler or passive BWS systems, using the standing position to adjust unloading force is the most practical option, although it may lead to a bias in the average unloading force.

\section{Viability of using simple gait models}

The model-predicted outcomes such as gait spatiotemporal parameters and ground reaction forces followed similar qualitative trends (increasing/decreasing) as the human data, despite high mrmse values for some parameters like the joint moments and muscle activities. This indicates that the response of gait models to BWS is akin to that of humans, albeit slightly exaggerated. The three gait models showed a stronger influence of BWS on most gait parameters than the experimental human data for both treadmill and overground walking conditions (Fig. 3 \& Appendix C - Fig. 6). While the human data presented a higher influence of BWS on the kinetic gait characteristics than on the gait spatio-temporal parameters and joint angles, the gait models also presented a larger effect for knee and ankle joint angle ROM, cadence, walking speed and double limb support phases. This was reflected in the higher mrmse for the cadence, walking speed and the joint angle ROM, as compared to the mrmse values for other spatio-temporal parameters, especially for the MR model. 
In case of the CF BWS strategy, the range of $\beta_{\max }$ values lied between $40 \%$ to $45 \%$ for the SLIP and MR gait models (Table 3). Above this range, the unloading force led to such a strong influence on the gait parameters that the gait models were unable to attain a walking gait. This range of $\beta_{\max }$ values aligns approximately with the $30 \%$ BWS level, up to which the influence of BWS on gait spatio-temporal and kinematic parameters has been shown to be limited [2, 12, 43-47].

For simulating higher BWS levels, it could be useful to refer to studies by Glauser et al. [18] and Ma et al. [20] which employ simulation techniques to predict the influence of BWS on human gait. The first study [18] employs a mass-spring-damper (MSD) system with two lumped masses representing the upper and the lower body, while the second study uses human motion capture data in conjunction with LifeMOD ${ }^{\mathrm{TM}}$ simulation package. However, it is necessary to note that the MSD model has not been validated against human data, while the second method is cumbersome and can be biased by the subject-specific nature of the motion capture data.

\section{Limitations of this study and potential directions for further research}

A major limitation of this study is that it only considers movement in the sagittal plane. It has been shown that BWS also impacts gait, particularly balance, in other planes $[48,49]$.

A possible extension of this study would be to consider other models suggested for gait, for example based on optimization [50,51], neural control and central pattern generators [52-54], the Virtual Pivot Point (VPP) [55], or the capture point [56]. Nonetheless, the selected gait models already cover most of the main features of human gait like mechanical stability, compliant nature of legs, segmented legs, muscle-reflex architecture, and the $\mathrm{m}$-shape of vertical GRF [57].

Cost of transport (COT) or metabolic cost for walking could be another measure to analyze when comparing gait models. It is known that COT decreases with the increase in BWS and that COT is an important governing factor for gait transitions [12]. Mechanical work could be calculated from the joint power consumption. However, while this work is correlated to the COT, it cannot be used to accurately determine the COT [58].

The point of application of the unloading force on the upper body may also play an important role. For the MR model, the $\beta_{\max }$ values for the TS and CF BWS strategies were highest if the unloading force was applied close to the COM of the upper body (Appendix B). This suggests that any moment of the unloading force on the upper body, even if the force is applied at a small distance from the upper-body COM, has a destabilizing effect. An indepth investigation of the behavior of gait characteristics for different locations of the BWS application point could be useful for the design of harness systems and for choosing between pelvic or body harness-based attachments. In case of the CW BWS strategy, the effective COM location changes due to the counterweight, thus making it difficult to predict the $\beta_{\max }$ behaviour.

In contrast to the predominant goal of constant, or unmodulated unloading force in active BWS, also modulated active BWS has been suggested, where the unloading force is controlled according to specific gait parameters. Recently, some interesting modulated BWS systems have been suggested [12], such as one that controls the unloading force based on gait cycle phases [37], another where the centre of pressure trajectory governs the unloading force [59], and a system that aims to dynamically compensate the inertial forces of the user's body [38]. It appears that the modulation of unloading force can facilitate appropriate ground contact and limb motion while allowing gait spatio-temporal parameters like walking speed, cadence and stride length to remain comparable to the values during unsupported walking [12]. Evidence still remains limited to pilot studies though.

Several BWS designs also allow for modulation of the force vector in other directions than the vertical $[13,41$, 42]. Appropriate interplay of vertical and forward forces may be another mechanism when striving for similar gait dynamics [31]. Simulation of gait models with modulated vertical and/or forward forces can provide the first step towards the detailed experimental studies for validating modulated BWS designs.

\section{Conclusion}

The primary goal of this research was to benchmark widely used gait models based on their suitability to the simulation of human walking with BWS. Gait models were simulated under the influence of Constant-Force, Counterweight, and Tuned-Spring BWS strategies. The results of this work strengthen the idea that reasonably simple gait models can be effectively used to simulate the effects of BWS on human locomotion. This study demonstrates the usefulness of gait models for BWS simulation, with the SLIP model having matched the human data more closely than the Simplest Walker and the Muscle-reflex models. However, the viability of gait models varies strongly with the type of BWS strategy and the initial gait speed. The results also point to limitations of the widely-used models in responding in a realistic way to external forces, indicating that they should be used only with caution outside of the situations they were developed, tuned, and evaluated for. Furthermore, the simulation results for the Tuned spring BWS strategy show promise and merit experimental investigation to compare its influence on human gait with that of a closed-loop control-based constant unloading strategy. 


\section{Appendix A: Implementation of TS BWS strategy} The Tuned-Spring (TS) BWS strategy is implemented using a first-order low-pass filter which is used to obtain the value of reference position of the spring, indicated by $y_{c 0}$ in Eq. 3. The rise time of this filter, from 0 to the value of the mean vertical position of the COM, is roughly 15 seconds. During initialization, the filter output is compared to the average vertical COM position at $0 \%$ BWS $(1.3037 \mathrm{~m})$ using an IF block, and the higher value among these two is used as the reference position $y_{c 0}$.

\section{Appendix B: Force application point for the MR model}

For the MR model, the selection of the location where the unloading force acts is an important decision. Since the limbs in this model are assumed to have mass, the center of mass of the body ( $\left.\mathrm{COM}_{\text {body }}\right)$ is different from the center of the mass of the upper body which includes the head, arms and trunk $\left(\mathrm{COM}_{\mathrm{HAT}}\right)$ and excludes the legs. The distance of the $\mathrm{COM}_{\text {body }}$ from the hip joint $(d)$, along the length of the upper body, was calculated using the $\mathrm{COM}_{\text {body }}$ position at three initial symmetric standing configurations: (1) legs at $90^{\circ}$ to horizontal (2) legs at $45^{\circ}$ to horizontal and (3) legs at $0^{\circ}$ to horizontal, a fictitious boundary case. The parameter $d$ was highest in the third case $(0.2341 \mathrm{~m})$ and so the $\beta_{\max }$ was computed at $d$ ranging from $0.23 \mathrm{~m}$ to $0.7 \mathrm{~m}, 0.7 \mathrm{~m}$ being two times the distance of $\mathrm{COM}_{\text {body }}$ from hip joint. The magnitude of $\beta_{\max }$ is highest typically around the position of the COM $\mathrm{CAT}_{\mathrm{HAT}}$ for $\mathrm{CF}$ and TS strategies, while it did not show a consistent behaviour

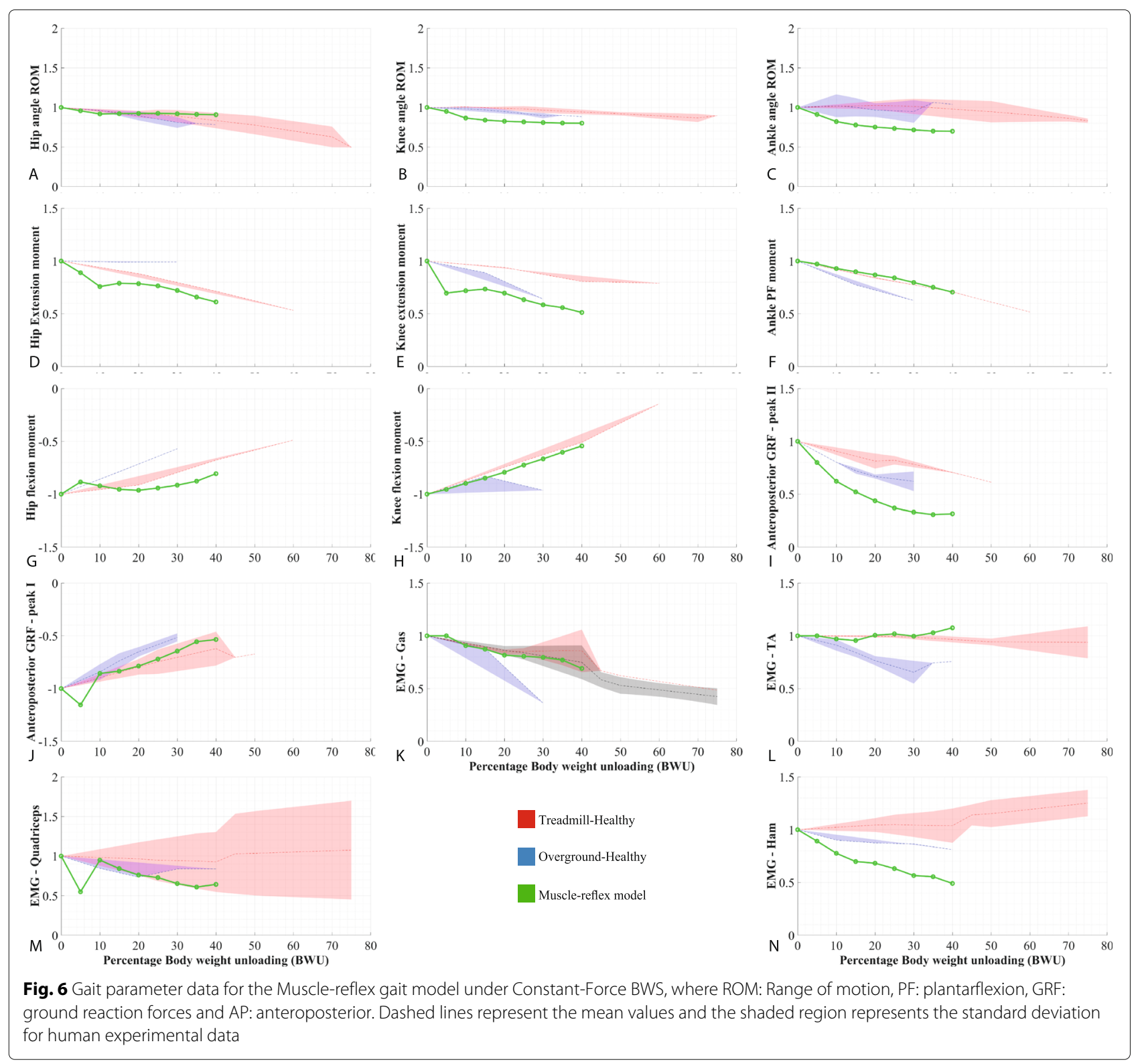


for the CW strategy. Thus, the $\mathrm{COM}_{\mathrm{HAT}}$ was chosen as the point of application of the unloading force since it is a well-defined point and leads to high $\beta_{\max }$ values.

\section{Appendix C: Additional results for gait parameters} Additional parameter trends are presented in Fig. 6. These only concern the MR model and hence do not allow comparison between the gait models. Joint dynamics, anteroposterior GRF and muscle activity plots are included here.

Table 5 Summary of the results of gait parameters for the MR model

\begin{tabular}{|c|c|c|c|c|c|c|}
\hline \multicolumn{4}{|c|}{ Root mean square error ( $m$-rmse) values } & \multirow{2}{*}{\multicolumn{3}{|c|}{ Treadmill (TM) }} \\
\hline \multirow{3}{*}{$\begin{array}{l}\text { Experimental } \\
\text { data } \\
\text { Gait model } \\
\text { Gait parameter }\end{array}$} & \multicolumn{3}{|c|}{ Overground (OG) } & & & \\
\hline & sw & SLIP & MR & \multirow{2}{*}{$\begin{array}{l}\text { Sw } \\
\%\end{array}$} & \multirow{2}{*}{$\begin{array}{l}\text { SLIP } \\
\%\end{array}$} & \multirow{2}{*}{$\begin{array}{l}\text { MR } \\
\%\end{array}$} \\
\hline & $\%$ & $\%$ & $\%$ & & & \\
\hline $\begin{array}{l}\text { 01. Hip joint - } \\
\text { ROM }\end{array}$ & - & - & 2.84 & - & - & 2.83 \\
\hline $\begin{array}{l}\text { 02. Knee joint - } \\
\text { ROM }\end{array}$ & - & - & 10.36 & - & - & 12.51 \\
\hline $\begin{array}{l}\text { 03. Ankle joint - } \\
\text { ROM }\end{array}$ & - & - & 18.27 & - & - & 18.61 \\
\hline $\begin{array}{l}\text { 04. Hip extension } \\
\text { moment }\end{array}$ & - & - & 18.85 & - & - & 13.46 \\
\hline $\begin{array}{l}\text { 05. Hip flexion } \\
\text { moment }\end{array}$ & - & - & 10.60 & - & - & 5.88 \\
\hline $\begin{array}{l}06 . \text { Knee } \\
\text { extension } \\
\text { moment }\end{array}$ & - & - & 21.49 & - & - & 25.47 \\
\hline $\begin{array}{l}\text { 07. Knee flexion } \\
\text { moment }\end{array}$ & - & - & 1.32 & - & - & 2.93 \\
\hline $\begin{array}{l}\text { 08. Ankle } \\
\text { plantarflexion } \\
\text { moment }\end{array}$ & - & - & 9.00 & - & - & 1.35 \\
\hline $\begin{array}{l}09 . \\
\text { Anteroposterior } \\
\text { GRF peak - I }\end{array}$ & - & - & 14.60 & - & - & 12.14 \\
\hline $\begin{array}{l}10 . \\
\text { Anteroposterior } \\
\text { GRF peak - II }\end{array}$ & - & - & 16.67 & - & - & 27.06 \\
\hline $\begin{array}{l}\text { 11. Muscle } \\
\text { activity - } \\
\text { Quadriceps }\end{array}$ & - & - & 22.60 & - & - & 26.73 \\
\hline $\begin{array}{l}\text { 12. Muscle } \\
\text { activity - } \\
\text { Hamstrings }\end{array}$ & - & - & 13.53 & - & - & 24.99 \\
\hline $\begin{array}{l}\text { 13. Muscle } \\
\text { activity - Medial } \\
\text { Gastrocnemius }\end{array}$ & - & - & - & - & - & 2.79 \\
\hline $\begin{array}{l}\text { 14. Muscle } \\
\text { activity - Lateral } \\
\text { Gastrocnemius }\end{array}$ & - & - & 2.47 & - & - & 2.52 \\
\hline $\begin{array}{l}\text { 15. Muscle } \\
\text { activity - Tibialis } \\
\text { anterior }\end{array}$ & - & - & 7.84 & - & - & 2.84 \\
\hline
\end{tabular}

Root mean square error ( $m$-rmse) values with respect to the experimental data for overground (OG) and treadmill (TM) environments are presented here

\section{Abbreviations}

BWS: Body weight support; SW: Simplest walking (gait model); SLIP: Spring-loaded inverted pendulum (gait model); MR: Muscle-reflex (gait model); ROM: Range of motion; GRF: Ground reaction forces; DLS: Double limb stance phase; SLS: Single limb stance phase

\section{Acknowledgements}

We would like to thank the authors of the original gait models and the authors of the Matlab implementations of these models. We would also like to thank Martijn Wisse for his valuable comments on the equations of motion of the

Simplest Walking model.

\section{Authors' contributions}

SA designed and conducted the simulations, analyzed and interpreted the data regarding the gait characteristics and was the main contributor to the manuscript. MP and $\mathrm{HV}$ checked and scrutinized the data, the analysis process and the results. In addition, MP and HV supervised the writing process for the manuscript. All authors read and approved the final manuscript.

\section{Funding}

Partially funded by Horizon 2020 Framework Programme, Award Number: 10 152, Recipient: Heike Vallery.

\section{Availability of data and materials}

The Matlab files used for simulation, along with the extracted gait data, are available at https://doi.org/10.4121/uuid:5899cdbf-e270-4a51-8d87c226b5fd80dd.

\section{Ethics approval and consent to participate}

Not applicable.

\section{Consent for publication}

Not applicable.

\section{Competing interests}

Heike Vallery and Michiel Plooij have been and continue to be involved in the design and commercialization of body-weight support systems.

\section{Author details}

${ }^{1}$ Department of Biomechanical Engineering, Delft University of Technology, Mekelweg 2, 2628 CD Delft, Netherlands. ${ }^{2}$ Motek Medical BV, Hogehilweg 18C, 1101 CD Amsterdam Netherlands. ${ }^{3}$ Laboratory of Movement Analysis and Measurement (LMAM), École Polytechnique Fédérale de Lausanne, Station 9 , CH-1015 Lausanne, Switzerland. ${ }^{4}$ Department of Rehabilitation Medicine, Erasmus MC, Postbus 2040, 3000 CA Rotterdam, Netherlands.

Received: 27 July 2019 Accepted: 21 May 2020

Published online: 25 June 2020

\section{References}

1. Hesse S, Bertelt C, Jahnke M, Schaffrin A, Baake P, Malezic M, Mauritz K. Treadmill training with partial body weight support compared with physiotherapy in nonambulatory hemiparetic patients. Stroke. 1995;26(6): 976-81.

2. Fischer AG, Wolf A. Assessment of the effects of body weight unloading on overground gait biomechanical parameters. Clin Biomech. 2015;30(5): 454-61.

3. Visintin M, Barbeau H, Korner-Bitensky N, Mayo NE. A new approach to retrain gait in stroke patients through body weight support and treadmill stimulation. Stroke. 1998;29(6):1122-8.

4. Schindl MR, Forstner C, Kern H, Hesse S. Treadmill training with partial body weight support in nonambulatory patients with cerebral palsy. Arch Phys Med Rehabil. 2000;81(3):301-6.

5. Miyai I, Fujimoto Y, Ueda Y, Yamamoto H, Nozaki S, Saito T, Kang J. Treadmill training with body weight support: its effect on parkinson's disease. Arch Phys Med Rehabil. 2000;81(7):849-52.

6. Barbeau H, Visintin M. Optimal outcomes obtained with body-weight support combined with treadmill training in stroke subjects. Arch Phys Med Rehabil. 2003;84(10):1458-65.

7. Hornby TG, Zemon DH, Campbell D. Robotic-assisted, body-weight-supported treadmill training in individuals following motor incomplete spinal cord injury. Phys Ther. 2005;85(1):52. 
8. Dietz V. Body weight supported gait training: from laboratory to clinical setting. Brain Res Bull. 2009;78(1).

9. Hicks AL, Ginis KM. Treadmill training after spinal cord injury: it's not just about the walking. J Rehab Res Dev. 2008;45(2):241.

10. Frey M, Colombo G, Vaglio M, Bucher R, Jorg M, Riener R. A novel mechatronic body weight support system. IEEE Trans Neural Syst Rehab Eng. 2006;14(3):311-21.

11. Sousa CO, Barela JA, Prado-Medeiros CL, Salvini TF, Barela AM. The use of body weight support on ground level: an alternative strategy for gait training of individuals with stroke. J Neuroeng Rehab. 2009;6(1):43.

12. Apte S, Plooij $M$, Vallery $H$. Influence of body weight unloading on human gait characteristics: a systematic review. J NeuroEng Rehab. 2018;15(1):53.

13. Nichols D. Zerog: overground gait and balance training system. J Rehab Res Dev. 2011;48(4):287.

14. Richter C, Braunstein B, Winnard A, Nasser M, Weber T. Human biomechanical and cardiopulmonary responses to partial gravity-a systematic review. Front Psychol. 2017. https://doi.org/10.3389/fphys. 2017.00583.

15. Haber F, Haber H. Possible methods of producing the gravity-free state for medical research. J Aviat Med. 1950;21:395-400.

16. Roberts JF. Walking responses under lunar and low gravity conditions. Technical report, AERONAUTICAL SYSTEMS DIV WRIGHT-PATTERSON AFB $\mathrm{OH} .1963$.

17. Vallery H, Duschau-Wicke A, Riener R. Hiding robot inertia using resonance. In: 2010 Annual International Conference of the IEEE Engineering in Medicine and Biology. IEEE; 2010. p. 1271-1274. https:// doi.org/10.1109/iembs.2010.5626416.

18. Glauser M, Lin Z, Allaire PE. Modeling and control of a partial body weight support system: an output regulation approach. IEEE Trans Control Syst Technol. 2010;18(2):480-90.

19. Ma O, Hernandez A, Liang J, Paz R. A testbed for testing an active body support system for locomotion training. In: Advanced Intelligent Mechatronics, 2008. AIM 2008. IEEE/ASME International Conference On. IEEE; 2008. p. 996-1001.

20. Lu Q, Liang J, Qiao B, Ma O. A new active body weight support system capable of virtually offloading partial body mass. IEEE/ASME Trans Mechatron. 2013;18(1):11-20.

21. Garcia M, Chatterjee A, Ruina A, Coleman M, et al. The simplest walking model: stability, complexity, and scaling. J Biomech Eng Trans ASME. 1998;120(2):281-8.

22. Geyer H, Seyfarth A, Blickhan R. Compliant leg behaviour explains basic dynamics of walking and running. Proc R Soc London B Biol Sci. 2006;273(1603):2861-7.

23. Geyer $\mathrm{H}$, Herr H. A muscle-reflex model that encodes principles of legged mechanics produces human walking dynamics and muscle activities. IEEE Trans Neural Syst Rehab Eng. 2010;18(3):263-73.

24. Alexander RM, Jayes A. A dynamic similarity hypothesis for the gaits of quadrupedal mammals. J Zool. 1983;201(1):135-52.

25. Vaughan $\mathrm{CL}, \mathrm{O}$ ?Malley MJ. Froude and the contribution of naval architecture to our understanding of bipedal locomotion. Gait Posture. 2005;21(3):350-62.

26. Donelan JM, Kram R. The effect of reduced gravity on the kinematics of human walking: a test of the dynamic similarity hypothesis for locomotion. J Exper Biol. 1997;200(24):3193-201.

27. Villeger D, Costes A, Watier B, Moretto P. Walking dynamic similarity induced by a combination of froude and strouhal dimensionless numbers: Modela-w. Gait Posture. 2015;41(1):240-5.

28. Apte S, Plooij M, Vallery H. Influence of body weight unloading on human gait characteristics: a systematic review [dataset]. TU Delft Dataset. 2018. https://doi.org/10.4121/uuid:6c42843c-6b07-4255-ad0f-7f6d8a994251.

29. Kajita S, Tani K. Study of dynamic biped locomotion on rugged terrain-theory and basic experiment. In: Advanced Robotics, 1991.'Robots in Unstructured Environments', 91 ICAR., Fifth International Conference On. IEEE; 1991. p. 741-6. https://doi.org/10.1109/icar.1991.240688.

30. Kuo AD. Energetics of actively powered locomotion using the simplest walking model. J Biomechanical Eng. 2002;124(1):113-20.

31. Mignardot J-B, Le Goff CG, Van Den Brand R, Capogrosso M, Fumeaux $\mathrm{N}$, Vallery H, Anil S, Lanini J, Fodor I, Eberle G, et al. A multidirectional gravity-assist algorithm that enhances locomotor control in patients with stroke or spinal cord injury. Sci Trans Med. 2017;9(399):3621.

32. Horchler AD. Passive-Dynamic-Walking. 2016. https://github.com/ horchler/Passive-Dynamic-Walking. Accessed 20 June 2017.
33. Saar KA. Bayesian Optimisation of SLIP model parameters. 2016. https://nl mathworks.com/matlabcentral/fileexchange/59060-bayesianoptimisation-of-slip-model-parameters?s_tid=prof_contriblnk. Accessed 10 June 2017.

34. Geyer H. A Muscle-Reflex Model That Encodes Principles of Legged Mechanics Produces Human Walking Dynamics and Muscle Activities. 2010. https://www.cs.cmu.edu/ hgeyer/Publications_Main.html. Accessed 25 Jul 2017

35. Perry J, Davids JR, et al. Gait analysis: normal and pathological function. J Pediatr Orthop. 1992;12(6):815.

36. Winter DA. Biomechanics and motor control of human movement: Wiley; 2009.

37. Franz JR, Glauser M, Riley PO, Della Croce U, Newton F, Allaire PE, Kerrigan DC. Physiological modulation of gait variables by an active partial body weight support system. J Biomech. 2007;40(14):3244-50.

38. Munawar H, Patoglu V. Gravity-assist: A series elastic body weight support system with inertia compensation. In: Intelligent Robots and Systems (IROS), 2016 IEEE/RSJ International Conference On. IEEE; 2016. p. 3036-41. https://doi.org/10.1109/iros.2016.7759470.

39. Cappellini G, Ivanenko YP, Poppele RE, Lacquaniti F. Motor patterns in human walking and running. J Neurophysiol. 2006;95(6):3426-37.

40. Geijtenbeek T. Scone: Open source software for predictive simulation of biological motion. J Open Source Softw. 2019;4:1421.

41. Vallery H, Lutz P, Von Zitzewitz J, Rauter G, Fritschi M, Everarts C, Ronsse R, Curt A, Bolliger M. Multidirectional transparent support for overground gait training. In: Rehabilitation Robotics (ICORR), 2013 IEEE International Conference On. IEEE; 2013. p. 1-7. https://doi.org/10.1109/ icorr.2013.6650512.

42. Plooij M, Keller U, Sterke B, Komi S, Vallery H, von Zitzewitz J. Design of rysen: An intrinsically safe and low-power three-dimensional overground body weight support. IEEE Robot Autom Lett. 2018;3(3):2253-2260.

43. Finch L, Barbeau H, Arsenault B, et al. Influence of body weight support on normal human gait: development of a gait retraining strategy. Phys Ther. 1991;71(11):842-55.

44. Threlkeld AJ, Cooper LD, Monger BP, Craven AN, Haupt HG Temporospatial and kinematic gait alterations during treadmill walking with body weight suspension. Gait Posture. 2003;17(3):235-45.

45. Van Hedel H, Tomatis L, Müller R. Modulation of leg muscle activity and gait kinematics by walking speed and bodyweight unloading. Gait Posture. 2006;24(1):35-45.

46. Field-Fote EC, Roach KE. Influence of a locomotor training approach on walking speed and distance in people with chronic spinal cord injury: a randomized clinical trial. Phys Ther. 2011;91(1):48-60.

47. Worthen-Chaudhari L, Schmiedeler JP, Basso DM. Training conditions that best reproduce the joint powers of unsupported walking. Gait posture. 2015;41(2):597-602.

48. Dragunas AC, Gordon KE. Body weight support impacts lateral stability during treadmill walking. J Biomech. 2016;49(13):2662-8.

49. Pennycott A, Wyss D, Vallery H, Riener R. Effects of added inertia and body weight support on lateral balance control during walking. In: Rehabilitation Robotics (ICORR), 2011 IEEE International Conference On. IEEE; 2011. p. 1-5. https://doi.org/10.1109/icorr.2011.5975415.

50. Anderson FC, Pandy MG. Dynamic optimization of human walking. J Biomech Eng. 2001;123(5):381-90.

51. Srinivasan $M$, Ruina A. Computer optimization of a minimal biped model discovers walking and running. Nature. 2006;439(7072):72-5.

52. Taga G. A model of the neuro-musculo-skeletal system for human locomotion. Biol Cybern. 1995;73(2):97-111.

53. Ogihara N, Yamazaki N. Generation of human bipedal locomotion by a bio-mimetic neuro-musculo-skeletal model. Biol Cybern. 2001;84(1):1-11.

54. Ijspeert AJ. Central pattern generators for locomotion control in animals and robots: a review. Neural Netw. 2008;21(4):642-53.

55. Maus H, Lipfert S, Gross M, Rummel J, Seyfarth A. Upright human gait did not provide a major mechanical challenge for our ancestors. Nat Commun. 2010;1(6):70.

56. Pratt J, Carff J, Drakunov S, Goswami A. Capture point: A step toward humanoid push recovery. In: Humanoid Robots, 2006 6th IEEE-RAS International Conference On. IEEE; 2006. p. 200-7. https://doi.org/10. 1109/ichr.2006.321385. 
57. Zajac FE, Neptune RR, Kautz SA. Biomechanics and muscle coordination of human walking: part ii: lessons from dynamical simulations and clinical implications. Gait Posture. 2003;17(1):1-17.

58. Umberger BR, Martin PE. Mechanical power and efficiency of level walking with different stride rates. J Exp Biol. 2007;210(18):3255-65.

59. Van ThuC T, Prattico F, Yamamoto S.-i. A novel treadmill body weight support system using pneumatic artificial muscle actuators: a comparison between active body weight support system and counter weight system. In: World Congress on Medical Physics and Biomedical Engineering, June 7-12, 2015, Toronto, Canada. Springer; 2015. p. 1115-9. https://doi.org/ 10.1007/978-3-319-19387-8_271.

\section{Publisher's Note}

Springer Nature remains neutral with regard to jurisdictional claims in published maps and institutional affiliations.

- fast, convenient online submission

- thorough peer review by experienced researchers in your field

- rapid publication on acceptance

- support for research data, including large and complex data types

- gold Open Access which fosters wider collaboration and increased citations

- maximum visibility for your research: over $100 \mathrm{M}$ website views per year

At $B M C$, research is always in progress.

Learn more biomedcentral.com/submissions 